\title{
Developing Positive Emotion through Affective Design for Interactive Information Seeking
}

\author{
Sehrish Sher Khan \\ University Of Bedfordshire \\ Institute for Research in Applicable Computing \\ Park Sqauare Luton \\ LU1 3JU, UK \\ sehrish.khan1@study.beds.ac.uk
}

\begin{abstract}
This research aims to convert the negative human emotional state to positive emotional state for better decision making in information seeking. A psychological emotional model will be used to identify the type of emotions. As emotions are triggers of decision making process in human and emotions are being influenced by the design of information seeking systems and the information overload, human behavior in information seeking is diverted to the negative emotional states due to design and information overload. Prior research has been focusing on specific type of emotion such as stress, frustration, anxiety in different scenarios. However, the gap is still there to not just only measure the type of emotion but to improve the affective wellbeing condition using the emotional data through improving the search system design. Design can heal or hurt. We would like to transform human negative emotional state to positive well-being by a better design approach in both human-computer interaction and human-human interaction.
\end{abstract}

Keywords: Emotion, Information Seeking, Behavior, Design, Positive, Well-Being.

\section{INTRODUCTION}

This paper aims at exploring the role of emotion in IR (Information Retrieval) and mainly in IS\&S (Information seeking and searching) processes. While the role of emotion in human behavior and activities has been extensively studied in the field of Psychology, Sociology and Physiology. Evidently, the role of emotion in computer science has been widely considered and being implemented to allow machines to behave and feel like humans. Affective needs are commonly known as emotion and feelings. In interactive information retrieval terms, affective need means users search experience and satisfaction. (Wilson 1997) Emphasized the fact that emotion, feelings and mood are the major motivator factors that motivate individuals for information accessing, seeking, and information using. The role of emotion, feelings and mood along with the cognitive approach allow the researchers to think about adapting the user-centered approach in information retrieval. This method is mainly concerned with the individual thinking, perception, behavior, feeling and emotions about information retrieval system and interaction process (McCracken $\&$ Wolfe 2004). A wide variety of study argued on the point that online search process is all related to human decision making. An association between unique patterns of emotional expressions and decision making has been investigated in the previous study to prove this point (Lopatovska 2011). The information is considered relevant to the users when it accommodates the users need to information. This support the point of decision-making regarding information use, the only information which is relevant would be utilized by the users.

The previous studies emphasized on considering the emotion as a secondary factor in any Information retrieval systems, searching and seeking processes (Savolainen 2015). Information seeking and searching is all related to human experience, human behavior, action and results. Emotion plays a significant role in influencing every aspect of human experience, behavior, action and their expressions. According to psychological theories, emotion converts into specific moods when lasts for more than an hour. The mood later changes the overall human behavior when lasts for days and weeks. In comparison, emotions and moods influence each other, if emotion is stronger, deep and crosses the threshold, it turns into either a good or bad mood (Hume 2012). Another study supports this statement 
that moods and other objectless affective states can be transformed into emotions (Clore \& Ortony 2000) (Ortony et al. 1990).

Previous studies focused on only analyzing the emotional searching and seeking behavior in Information Retrieval and lacks in providing the procedure that would further help individuals to change their negative emotional state to positive emotional state which will modify the mood and behavior.

Emotions are being influenced by the external and internal factors which cause to effect decisionmaking and overall human behavior. Those factors also include the overloading of information. Information is everywhere in the form of information overload, for this reason, we aim at targeting to investigate the inlfuence of information overload on human emotion and decision-making. To discuss all these issues, this research focuses on three main parts. 1) Emotional, psychological theory to be applied in the information searching and seeking experiences to detect individual emotional episodes. 2) Designing and developing the affective information searching system, 3) Changing the human behavior regarding positive emotional state using psychological, behavioral therapy adapting in design approaches. However, this research will focus on one of the emotional, psychological theory to investigate the human emotional states during information searching and seeking process. Moreover, a behavioral therapy will be studied based on the emotional searching and seeking results that help to change the human behavior based on the emotional search experiences, emotional search expressions and emotional search actions. Our aim is at designing and developing the affective information searching system Which can be adapted to the general scenario to enable positive emotions and well-being. The second part of the paper explains related work about emotion and information retrieval, third part explains the overall view of the research methodology to be adapted and last part explains provide the conclusion.

\section{BACKGROUND}

\subsection{Emotion And Information Retrieval}

Emotions, change the way the human mind solves problems and the emotional system changes how the cognitive system operates. Emotions are the key factor in any decision-making process. Emotion influence the searchers tasks, performance and satisfaction during information seeking process. Wilson in 1993 considered the role of affect and defined Affective need as an independent need which can motivate Information Retrieval and seeking behavior(Wilson 1997).

The information searcher is considered as an agent in this scenario searching and seeking for the required information while interacting with IR systems. Wilson introduced the correlation between the physiological, affective and information needs in Information retrieval and searching (Wilson 1997). Similarly, Kuhlthaus uncertainty principle and Nahls affective load theory (Kuhlthau 1993, Nahl 2005). These studies have investigated the role of affective and cognitive experience of a searcher in information searching and seeking process.

Moreover, the interest growing gradually in the research area to provide a theoretical study of affective factors in information seeking and information use (Nahl 1998a). These study shares the common phenomena of emphasizing the fact that emotion, feelings and mood are the major motivator factors that motivate individuals for information accessing, seeking, and information using (Nahl 1998b). Further, the previous studies focused on the understanding of users thoughts and their influence on their actions and affective state while interacting with information retrieval systems (Dervin 1983, Kuhlthau 1993, Ingwersen 1996).

Further, negative feelings were associated mainly with ideas related to the system, search strategy and tasks. Searching behavior is also associated with the learning styles of graduate students. The more the students get searching faster without pausing, the more they understand the search process (Tenopir et al. 2008). Also, (Arapakis et al. 2008) recognized the importance of user feedback as a critical element in information seeking process. Also, suggests that emotion is linked not only with different physiological, psychological and cognitive processes but also form a pattern with particular task and user (Arapakis et al. 2008).

An association between unique patterns of emotional expressions and decision making has also been investigated that helped researcher to explore the emotional aspects of information retrieval systems. Later, it helped them to develop affective IR systems that are capable of recognizing and responding to users emotions (Lopatovska 2011).

\section{RESEARCH FOCUS}

The users interaction when relates to system in terms of information seeking and searching behavior requires attention. Introducing the cognitive theory in information interaction one way or another attempt 
to explain the role of human feelings in information seeking and searching behavior.

Previous studies ignored one of the principals of psychological emotion theories is the way human emotion changes into moods and behavior. Researcher focused only on the emotion detection, while emotion lasts only for seconds and minutes.Our aim is investigating the emotional data in a time-line manner with specific moods and behaviors using a psychological emotion theory (Arnold 1960). This theory provides us the better overview of the emotional states we need to investigate in the research. This has been the widest used theory in human phsychology. This would help us to gain the required human experiences in physiological responses, human expressions either verbal or non-verbal and user's actions. This theory is best suited for our research.

Secondly, design and Development of an Affective Search System, to accommodate the affective information searching needs and behavior to change the negative emotional search state to positive emotional search state. Later, which can be adapted to general scenario to enable positive emotions and well-being. Next, finding the influence of external factor called information overload on emotion, information searching and human behavior. This would help us to find the relationship between these key concepts.

Finally, changing human behavior through design approaches has also been not discussed in the previous studies. The psychological behavioral change could be applied after detecting emotions with help of psychology theory to change and identify the factors that causes to change the human behavior (Corstorphine 2006).

\section{RESEARCH METHODOLOGY}

To assess emotions is not an easy task and there is no conclusive study to follow to conduct our experiments. Our study will be the first to use these types of hardware to assess user emotions in the context of Information Seeking. The Below hardware's will be utilized in this study.

\subsection{Hardwares}

\subsubsection{Shimmer3 Galvanic Skin Response Sensor}

This device captures real time change in user behavior. It captures any stimulus caused in the environment of the user through the user skin. The responses from this captured data will interpret into the following emotion, e.g., stress, excitement, Fear, Sadness, Joy, Anger, Trust, Anticipation.

\subsubsection{Emotive insight EEG Headset}

This device will capture any emotional changes occurred in the human brain. The collected data in the form of EEG (electroencephalography) will categorize into the following emotions, e.g., stress, excitement, Fear, Sadness, Joy, Anger, Trust, Anticipation.

The obtained data from both devices will be synchronized to achieve maximal results and validate the outcomes from the data.

\subsection{Research Strategy}

The research includes three stages research strategy. At the first phase, our aim is at implementing the emotional psychology theory to identify the emotional experience, emotional expressions and emotional actions as depicted in Figure 1. These three key emotion psychological points are being adapted from (Arnold 1960) theory. Arnold's theory is one of the widest used theory in Psychology, using this theory would help us to identify the emotional experiences in terms of physiological responses, emotional expressions and emotional actions taken by search system users. Aim is at identifying the changes in mood with respect to emotion changes in a time-line manner. Also, with the help of this methodology, our aim is investigating the effect of information overload and its role in influencing affective information searching and seeking behavior in affective information system design.

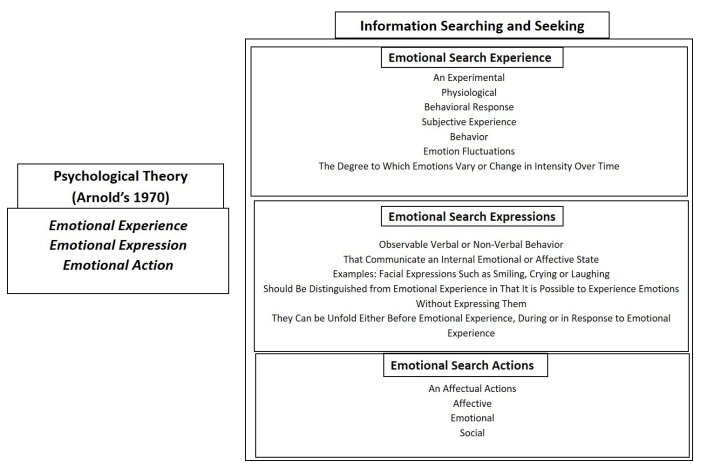

Figure 1: Psychological Theory to Be Implemented in Information Searching and Seeking (Arnold 1960)

The main design strategy that would work as a trigger is the affective design which will be developing by starting from small affective design approach in $\mathrm{HCl}$ for affective information search system design. At this phase, our aim is designing and developing of the affective search system design and its modification according to the experiments and user's affective needs and scenarios.

Figure 2 depicts the cognitive behavioral therapy to be adapted in computational research. 


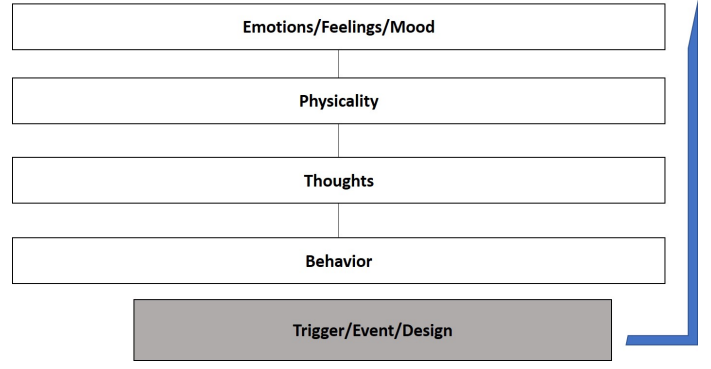

Figure 2: Physchological Cognitive Behaviorial Therapy to be Adapted in the Research

\section{CONCLUSION}

This research will highlight the role of emotion in major issues related to information retrieval, information seeking and searching behavior, the affective information need, the affective behavior, affective design and affective action. Main motivation of the research is to change the individuals negative emotional state to positive emotional state using the interactive affective information searching scenarios and systems. This motivation could be effective in terms of information searching and seeking behavior while interacting with the term information overload.

To achieve the research target, we are considering emotion theory and design and emotion established practices to determine a reflective analysis of how to design for individual positive emotional state in terms of information seeking and searching processes. These considerations include how human behavior is changed because of emotions elicited by certain products and explained from a psychology perspective and by using a psychological therapy to change the behavior to positive. Further, design and development of an affective search system can be later adapted to general scenario to enable positive emotions and well-being

\section{REFERENCES}

Arapakis, I., Jose, J. M. \& Gray, P. D. (2008), Affective feedback: an investigation into the role of emotions in the information seeking process, in 'Proceedings of the 31st annual international ACM SIGIR conference on Research and development in information retrieval', ACM, pp. 395-402.

Arnold, M. B. (1960), 'Emotion and personality.'.

Clore, G. L. \& Ortony, A. (2000), 'Cognition in emotion: Always, sometimes, or never', Cognitive neuroscience of emotion pp. 24-61.

Corstorphine, E. (2006), 'Cognitiveemotionalbehavioural therapy for the eating disorders: Working with beliefs about emotions', European Eating Disorders Review 14(6), 448-461.
Dervin, B. (1983), An overview of sense-making research: Concepts, methods, and results to date, The Author.

Hume, D. (2012), A treatise of human nature, Courier Corporation.

Ingwersen, P. (1996), 'Cognitive perspectives of information retrieval interaction: elements of a cognitive ir theory', Journal of documentation 52(1), 3-50.

Kuhlthau, C. C. (1993), 'Seeking meaning', Norwood, NJ: Ablex.

Lopatovska, I. (2011), Emotional correlates of information retrieval behaviors, in 'Affective Computational Intelligence (WACI), 2011 IEEE Workshop on', pp. 1-7. ID: 1.

McCracken, D. D. \& Wolfe, R. J. (2004), Usercentered website development: A humancomputer interaction approach, Prentice Hall Englewood Cliffs.

Nahl, D. (1998a), 'Ethnography of novices' first use of web search engines: affective control in cognitive processing', Internet Reference Services Quarterly 3(2), 51-72.

Nahl, D. (1998b), 'Learning the internet and the structure of information behavior', Journal of the American Society for Information Science 49(11), 1017-1023.

Nahl, D. (2005), 'Affective and cognitive information behavior: Interaction effects in internet use', Proceedings of the American Society for Information Science and Technology 42(1).

Ortony, A., Clore, G. L. \& Collins, A. (1990), The cognitive structure of emotions, Cambridge university press.

Savolainen, R. (2015), 'Expressing emotions in information sharing: a study of online discussion about immigration.', Information Research 20(1).

Tenopir, C., Wang, P., Zhang, Y., Simmons, B. \& Pollard, R. (2008), 'Academic users interactions with sciencedirect in search tasks: Affective and cognitive behaviors', Information Processing Management 44(1), 105-121.

Wilson, T. D. (1997), 'Information behaviour: an interdisciplinary perspective', Information processing management 33(4), 551-572. 